\title{
Three-dimensional High-frequency Spectral and Envelope Quantification of Excised Human Lymph Nodes
}

\author{
Jonathan Mamou*†, Alain Coron ${ }^{\ddagger \S}$, Michael L. Oelze\#, Emi Saegusa-Beecroft" ${ }^{\|}$, Masaki Hata", \\ Junji Machill, Eugene Yanagihara ${ }^{\|}$Pascal Laugier ${ }^{\ddagger \S}$ and Ernest J. Feleppa ${ }^{\dagger}$ \\ ${ }^{\dagger}$ Frederic L. Lizzi Center for Biomedical Engineering, Riverside Research Institute, New York, NY, USA \\ $\S$ UPMC Univ Paris 06, UMR 7623, LIP, F-75005, Paris, France

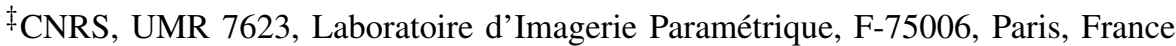 \\ \# Bioacoustics Research Laboratory, University of Illinois, Urbana, IL, USA \\ "University of Hawaii and Kuakini Medical Center, Honolulu, HI, USA \\ *E-mail: jmamou@rri-usa.org
}

\begin{abstract}
Quantitative imaging methods using high-frequency ultrasound (HFU, $>20 \mathrm{MHz}$ ) offer a means of characterizing biological tissue at the microscopic level. In this study, threedimensional (3D) quantitative-ultrasound (QUS) methods were developed to detect metastases in freshly-dissected lymph nodes of cancer patients. 3D ultrasound radio-frequency data were acquired using a 26-MHz center-frequency transducer, and each node was inked prior to tissue fixation to recover orientation after sectioning for 3D histological evaluation. Backscattered echo signals were processed using 3D cylindrical regions-ofinterest (ROIs) to yield eight QUS estimates associated with tissue microstructure. The first four QUS estimates (i.e., effective scatterer size, acoustic concentration, intercept, and slope) were estimated from a normalized backscattered spectrum, and the other four QUS estimates were obtained by parameterizing the envelope statistics of the ROIs using Nakagami and homodynedK models. These QUS methods were applied to 145 lymph nodes from 95 colorectal and gastric cancer patients. The ability of these eight QUS estimates to classify lymph nodes and detect cancer was evaluated using ROC curves. An area under the ROC curve of 0.971 with specificity and sensitivity of $91 \%$ (using a leave-one-out procedure) were obtained by combining effective scatterer size and one envelope parameter based on the homodyned-K distribution. Therefore, these advanced 3D QUS methods potentially can be valuable for detecting small metastatic foci in dissected lymph nodes.
\end{abstract}

Index Terms-high-frequency ultrasound, quantitative ultrasound, lymph node.

\section{INTRODUCTION}

Conventional B-mode images have a resolution on the order of a wavelength; therefore, high-frequency (i.e., >20 MHz) ultrasound (HFU) permits imaging with exquisite resolution (e.g., $<100 \mu \mathrm{m}$ ). However, HFU imaging suffers from two main drawbacks: first, the depth of field of typical HFU images is decreased because such systems use single-element transducers with small F-numbers, and second, penetration depth of HFU is more limited because of frequency-dependent attenuation. Nevertheless, many studies in the past 20 years have demonstrated the unique ability of HFU systems to image shallow or low-attenuation tissues for biomedical applications with fine resolution. For example, recent HFU studies have shown success for small-animal [1], ocular [2], and dermatological imaging [3].

In another recent study, our group demonstrated the ability of three-dimensional (3D) quantitative ultrasound (QUS) to characterize lymph-node tissues from cancer patients [4]. Reliable determination of the presence or absence of metastatic cancer in lymph nodes is essential for staging disease and planning its treatment. Most human lymph nodes have sizes ranging from 2 to $10 \mathrm{~mm}$ and are sufficiently small to be imaged in their entirety in 3D using HFU. In routine pathology procedures, our QUS methods would be used to direct the pathologist to suspicious nodal regions that might be overlooked in conventional histology. To achieve this objective, previous QUS studies that were undertaken successfully [4] have been improved, including envelope statistics, and applied to a larger number of lymph nodes to enhance statistical significance.

The present study focuses on two different categories of QUS methods. The first category quantifies the backscattered spectrum deduced from the radio-frequency (RF) echo signals [4]-[7] and the second category quantifies the statistics of the envelope-detected echo signals. In the second category, the QUS estimates that are derived from fit parameters associated with the envelope hypothetically provide an additional means of distinguishing among tissue types and complement the QUS estimates from the first category that are derived from backscattered RF signals [8]. In this study, the Nakagami and the more-complex homodyned-K $(\mathrm{HK})$ distribution were used to obtain four additional QUS estimates [9].

\section{MethodS}

Lymph nodes were dissected from patients with histologically-proven colorectal and gastric cancers at the Kuakini Medical Center (KMC) in Honolulu, HI. Then, 
individual, manually-defatted, fresh lymph nodes were placed in a saline bath at room temperature and scanned individually. Ultrasound data were acquired with a focused, single-element transducer (PI30-2-R0.50IN, Olympus NDT, Waltham, MA) with an aperture of $6.1 \mathrm{~mm}$ and a focal length of $12.2 \mathrm{~mm}$. The transducer had a center frequency of $25.6 \mathrm{MHz}$ and a $-6 \mathrm{~dB}$ bandwidth that extended from 16.4 to $33.6 \mathrm{MHz}$. The transducer was excited by a Panametrics 5900 (Olympus NDT, Waltham, MA) pulser/receiver unit. The RF echo signals were digitized at $400 \mathrm{MHz}$. The spacing between adjacent A-lines was $25 \mu \mathrm{m}$. RF data were acquired in 3D by scanning adjacent planes every $25 \mu \mathrm{m}$ to uniformly cover the entire lymph node. A semiautomatic 3D segmentation method was implemented in MATLAB (The MathWorks, Inc., Natick, MA) to separate lymph-node tissue from surrounding fibroadipose tissue and saline [10]. Then, the complete 3D RF data set was separated into overlapping 3D cylindrical ROIs having a diameter of $1 \mathrm{~mm}$ and a length (i.e., along the axis of the transducer) of $1 \mathrm{~mm}$ [4]. Estimates were computed for each ROI only if the ROI was entirely contained in the lymph-node tissue region determined using the $3 \mathrm{D}$ segmentation algorithm.

Following ultrasound scanning, lymph nodes were inked to provide visible references for subsequent reassembly of histology into 3D volumes and spatial matching with volumes generated from QUS processing. Then, the node was cut longitudinally in half and the two half-nodes were fixed in $10 \%$ neutral-buffered formalin and embedded in paraffin with the flat cut surface oriented downward in the embedding cassette. Following fixation, the two half-nodes were sectioned and stained with hematoxylin and eosin (H\&E). Thin sections were prepared at $50 \mu \mathrm{m}$ intervals for nodes smaller than $5 \mathrm{~mm}$ in maximum dimension and at $100 \mu \mathrm{m}$ intervals for nodes larger than $5 \mathrm{~mm}$ in maximum dimension. This histological approach assured detection of small micrometastases $(0.2-\mathrm{mm}$ to 2-mm in size). Finally, the microscope slides containing the H\&E-stained thin sections were evaluated by a board-certified pathologist and the pathologist identified and demarcated the border of each detected metastases in the examined thin sections.

The methods to estimate the four QUS parameters based on the backscatter spectrum for each 3D ROI have been described in great detail previously [4] and have not been modified. The four new envelope-based QUS parameters were computed by fitting distribution models to the envelope statistics of each 3D ROI. The first two parameters $\alpha$ and $\Omega$ were obtained using a maximum-likelihood estimator to fit a Nakagami probability density function (PDF) to that of the envelope. The parameter $\alpha$ is called the Nakagami parameter and can be related to the number of scatterers per resolution cell.

Two additional QUS parameters, $k$ and $\mu$, were obtained using the HK distribution to model the envelope statistics within the 3D ROI. The algorithm estimated envelope-statistics parameters by calculating the signal-to-noise ratio (SNR), skewness, and kurtosis of fractional-order moments of the envelope samples in each ROI. The optimal pair of fractional- order moments (i.e., 0.72 and 0.88 ) was found by calculating the maximal intersection angles between the six level curves generated at each pair of $k$ and $\mu$ values over a range of values expected to be encountered in ultrasonic imaging [9]. Level curves previously generated and stored for the SNR, skewness, and kurtosis corresponding to different values of the $k$ and $\mu$ parameters for the two fractional-order moments were used to find the intersection of the six level curves generated from the envelope values from the ROI in the $k-\mu$ plane, and yielded the estimated values of $k$ and $\mu$. The parameter $\mu$ represents the number of scatterers per resolution cell and $k$ is the ratio of coherent to incoherent energy.

\section{RESUlts}
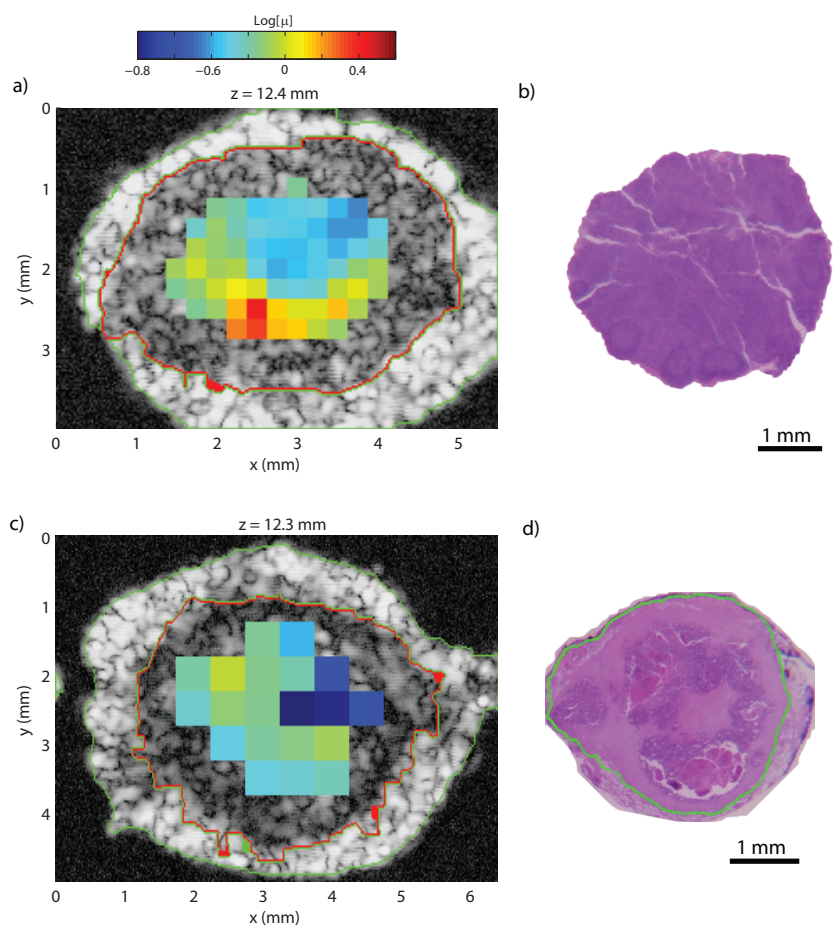

d)

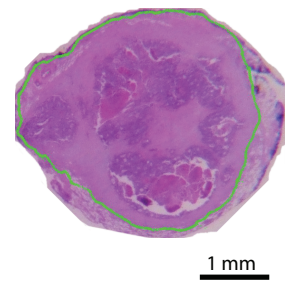

Fig. 1: Illustrative results obtained with a non-metastatic lymph node ( $a$ and $b$ ) and a nearly entirely metastatic lymph node (c and d). a) and c): parametric cross-sectional images displaying effective $\log (\mu)$ estimates. b) and d) histologic thin section approximately corresponding to a) and c), respectively. Metastatic region is highlighted in green in d) and segmentation results are shown by the green and red highlights in a) and $\mathrm{c}$ ).

Figure 1 displays illustrative results obtained from two lymph nodes from two patients diagnosed with colon cancer. Figures $1 \mathrm{a}$ and $1 \mathrm{~b}$ display a parametric image from $\log (\mu)$ at a fixed depth near the transducer focus and the photomicrograph of the approximately corresponding histologic thin section. The histology revealed that this lymph node was non metastatic. Similarly, Figs. 1c and 1d display the images for a metastatic lymph node. The pathologist indicated that this lymph node was almost entirely metastatic by the green 
TABLE I: Average QUS estimates (means \pm standard deviations) for the non-metastatic and metastatic nodes. (The symbol "*” indicates statistical significance based on ANOVA results giving $p<0.05$.)

\begin{tabular}{|c|c|c|}
\hline QUS estimate & Non-metastatic $(N=122)$ & Metastatic $(N=23)$ \\
\hline$D(\mu \mathrm{m})$ & $28.9 \pm 3.3^{*}$ & $36.5 \pm 2.6^{*}$ \\
$\left.C Q^{2}(\mathrm{~dB} \mathrm{~mm})^{-3}\right)$ & $-3.9 \pm 2.41^{*}$ & $-7.92 \pm 4.20^{*}$ \\
\hline$I(\mathrm{~dB})$ & $-63.1 \pm 3.9^{*}$ & $-58.1 \pm 4.5^{*}$ \\
$S(\mathrm{~dB} / \mathrm{MHz})$ & $0.30 \pm 0.12^{*}$ & $0.02 \pm 0.11^{*}$ \\
\hline $\log [\alpha]$ & $-0.26 \pm 0.05^{*}$ & $-0.32 \pm 0.07^{*}$ \\
$\log [\Omega]\left(\mathrm{dB} \mathrm{V}^{2}\right)$ & $-6.55 \pm 0.21$ & $-6.64 \pm 0.38$ \\
\hline $\log [\mu]$ & $-0.11 \pm 0.18^{*}$ & $-0.35 \pm 0.23^{*}$ \\
$k$ & $0.56 \pm 0.13$ & $0.57 \pm 0.10$ \\
\hline
\end{tabular}

highlight shown in Fig. 1d. For each lymph node, the histology and the parametric images are on the same scale for easier comparison, and segmentation results are shown in Figs. 1a and $1 \mathrm{c}$ by the green and red highlights. The green and red highlights surround the fat and lymph-node tissue, respectively. Figures 1a and 1c show limited contrast in the $\log (\mu)$ estimates. The same color scale was used for both images. The metastatic lymph node displays slightly smaller values. The average $\log (\mu)$ estimates for each entire lymph node were $-0.14 \pm 0.22$ for the non-metastatic node and $-0.34 \pm 0.22$ for the metastatic node. The large variations in the estimates of $\log (\mu)$ illustrate how differentiating a metastatic node from a non-metastatic node potentially would be possible, but difficult using $\mu$ alone. Typical cross-section images of other QUS parameters have been published previously and, in particular, scatterer-size images demonstrated significant potential for diagnosis and cancer localization [4].

The statistics of the four QUS estimates based on backscatter quantification are in strong agreement with our previously published results derived from a significantly lower number of lymph nodes [4]. The trend observed in Table I indicates that metastatic nodes have significantly higher effective scatterer-size estimates (i.e., $D$ ) and higher intercept estimates (i.e., $I$ ), and significantly lower slope (i.e., $S$ ) and acousticconcentration estimates (i.e., $C Q^{2}$ ). The other four QUS estimates were obtained from the envelope statistics and the two distribution models (Table I). No statistically significant differences were observed between the cancer-free and cancerfilled nodes for $\log (\Omega)$ and $k$, but estimates of $\log (\alpha)$ and $\log (\mu)$ were significantly lower in the metastatic lymph nodes. This is consistent with the physical interpretation of $\mu$ and $\alpha$ being related to scatterer number density. Metastatic nodes, which have larger effective scatterer-size estimates than nonmetastatic nodes, are likely to have a concomitantly lower number density. Note that although statistically significant differences were observed for $\mu$ and $\alpha$, the standard deviations of the estimates were fairly large and significant overlap existed between the estimates obtained between the two types of nodes.

To further quantify these observations, the software package SPSS (SPSS Inc., Chicago, IL) was used to generate ROC curves for each individual QUS estimate and for several com- binations of the eight QUS estimates using linear-discriminant analysis (Table II). SPSS also was employed to evaluate classification performance using a leave-one-out procedure and the resulting specificity and sensitivity were computed (Table II). Looking first at the numbers obtained with the four QUS estimates quantifying backscatter power spectra, the results indicate that using $D$ alone, excellent classification performance can be achieved with an area under the ROC curve (AUC) of 0.964 . Specificity and sensitivity also were excellent with both values above $88 \%$. The moderate overlap existing among the acoustic-concentration values produced an AUC value of 0.830 , a specificity of $80 \%$, and a sensitivity of $74 \%$; therefore, acoustic-concentration estimates alone would classify lymph nodes only moderately well. Finally, combining $D$ and $C Q^{2}$ only marginally improves classification performance over $D$ alone (AUC value of 0.970). $I$ alone performs moderately well as does $C Q^{2}$ alone, and $S$ performs well with a performance only slightly inferior to $D$ alone. Finally, combining $I$ and $S$ together produces an AUC value of 0.955 , with sensitivity and specificity values above $87 \%$.

Results for the other four QUS estimates based on envelope statistics indicate that diagnostic performance using any of these estimates alone would be unsatisfactory. AUC values obtained from $\Omega$ or $k$ were smaller than 0.6. The Nakagami parameter, $\alpha$, performed significantly better, but it still produced a mediocre AUC value of 0.759 . Finally, the best performance was obtained using $\mu$ leading to an AUC value of 0.797 , with sensitivity and specificity values near $70 \%$. Interestingly, combining the Nakagami model parameters, $\alpha$ and $\Omega$, led to an AUC value of 0.826 , meaning that the Nakagami envelope model would classify lymph nodes moderately well. However, combining the two HK model parameters, $k$ and $\mu$, did not improve classification performance over $\mu$ alone. Nevertheless, the HK and Nakagami model classification performance only remains moderately satisfactory. These results indicate that the HK model produced the best QUS estimate (i.e., $\mu$ ) for classification based on envelope quantification, but they also indicate that the Nakagami model slightly outperformed the HK model when the two independent QUS estimates from each model were combined.

Finally, the best classification results were obtained by combining $D$ and $k$, which led to an AUC value of 0.971 and sensitivity and specificity values around $92 \%$. (No other combination of two or more QUS estimates led to a better performance.) To illustrate how linear-discriminant analysis can be used for the detection of cancerous regions, we computed a posteriori cancer probability using the linear-disciminant function obtained from $D$ and $k$. This function was used to highlight in red in $3 \mathrm{D}$, the regions where cancer likelihood was estimated to be greater than $50 \%$ in a partially-metastatic lymph node (Fig. 2). Figure 2 shows agreement between the QUS-derived cancerous region and the demarcated region on the histology photomicrograph. Such an approach would be straightforward to use clinically and would provide an easy means to guide pathologists towards suspicious regions. 


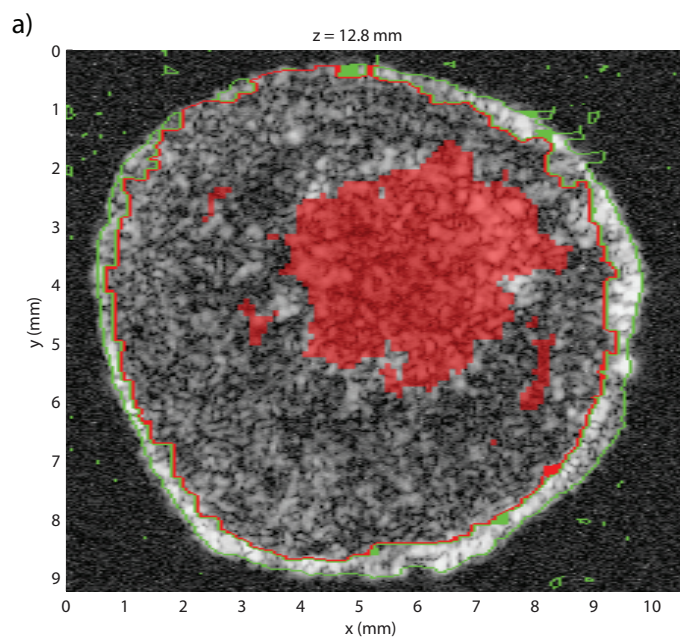

b)

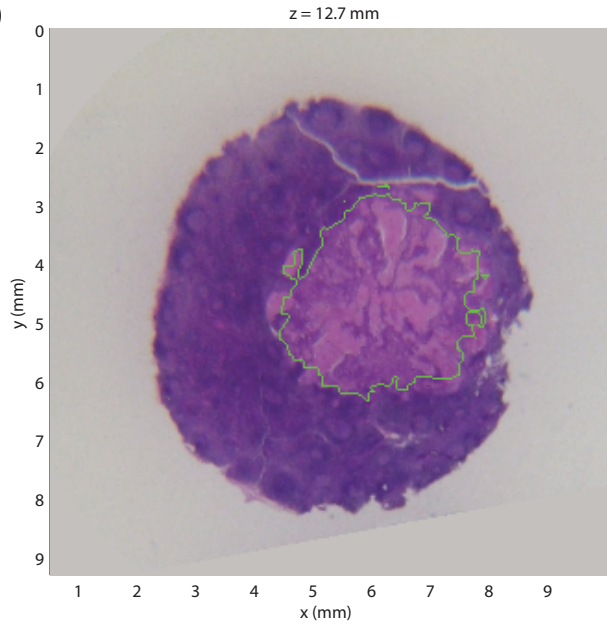

Fig. 2: Partially-metastatic lymph node from a colorectalcancer patient. a) Regions highlighted in red indicate a $a$ posteriori cancer probability greater than $50 \%$. b) Histologic thin section approximately corresponding to a).

\section{CONCLUSIONS AND PERSPECTIVES}

In this study, previously-developed 3D QUS methods were refined to include envelope statistics and applied to freshlyexcised human lymph nodes scanned using a custom HFU system. 3D ultrasound data and histologic data were obtained and spatially matched for 145 freshly-excised lymph nodes from patients diagnosed with primary colorectal or gastric cancer. A total of eight QUS parameters were estimated for each ROI within each lymph node. The ability of these parameters to detect metastatic tissue was carefully studied using lineardiscriminant analysis. Results were satisfactory, and excellent classification performance was obtained when scatterer size and an envelope parameter were used for classification (i.e., producing areas under the ROC curve greater than 0.97). The results also indicated how these QUS methods could be used in the clinic by spatially outlining suspicious regions within a lymph node. Therefore, these methods could provide reliable specific localization of micrometastases, which is crucial for
TABLE II: Classification performance of QUS. Areas under the ROC curve (AUC) and $95 \%$ confidence interval are presented. Sensitivity and specificity were obtained using lineardiscriminant analysis with a leave-one-out procedure.

\begin{tabular}{|c||c|c|c|c|}
\hline QUS Estimates & ROC AUC & 95\%-Confidence & Sensitivity & Specificity \\
\hline$D$ & 0.964 & $0.933-0.995$ & $91.3 \%$ & $88.5 \%$ \\
$C Q^{2}$ & 0.830 & $0.730-0.930$ & $73.9 \%$ & $80.3 \%$ \\
$D$ and $C Q^{2}$ & 0.970 & $0.944-0.996$ & $87.0 \%$ & $89.3 \%$ \\
\hline$I$ & 0.805 & $0.701-0.909$ & $73.9 \%$ & $72.1 \%$ \\
$S$ & 0.954 & $0.920-0.988$ & $87.0 \%$ & $88.5 \%$ \\
$I$ and $S$ & 0.955 & $0.922-0.989$ & $87.0 \%$ & $88.5 \%$ \\
\hline $\log [\alpha]$ & 0.759 & $0.642-0.879$ & $60.9 \%$ & $71.3 \%$ \\
$\log [\Omega]$ & 0.570 & $0.435-0.705$ & $56.5 \%$ & $59.0 \%$ \\
$\log [\alpha]$ and $\log [\Omega]$ & 0.826 & $0.717-0.935$ & $69.6 \%$ & $74.6 \%$ \\
\hline$k$ & 0.520 & $0.393-0.648$ & $56.5 \%$ & $44.3 \%$ \\
$\log [\mu]$ & 0.797 & $0.684-0.909$ & $69.6 \%$ & $75.4 \%$ \\
$k$ and $\log [\mu]$ & 0.797 & $0.684-0.909$ & $69.6 \%$ & $75.4 \%$ \\
\hline$D$ and $k$ & 0.971 & $0.943-0.999$ & $91.3 \%$ & $92.6 \%$ \\
\hline
\end{tabular}

staging and treatment planning. Currently, the likelihood of missing small metastases is unacceptably high using conventional histology methods.

\section{ACKNOWLEDGEMENTS}

This research was supported in part by NIH Grant CA100183 and the Riverside Research Institute Fund for Biomedical Engineering Research. The authors wish to thank Paul Lee, Helena Herman, and Justin Rhoda for help with the 3D segmentation.

\section{REFERENCES}

[1] J. Mamou, O. Aristizabal, R. H. Silverman, J. A. Ketterling, and D. H. Turnbull, "High-frequency chirp ultrasound imaging with an annular array for ophthalmologic and small-animal imaging," Ultrasound Med Biol, vol. 35, no. 7, pp. 1198-208, 2009.

[2] R. H. Silverman, J. A. Ketterling, J. Mamou, and D. J. Coleman, "Improved high-resolution ultrasonic imaging of the eye," Arch Ophthalmol., vol. 126, pp. 94-97, 2008.

[3] Y. P. Huang, Y. P. Zheng, S. F. Leung, and A. P. Choi, "High frequency ultrasound assessment of skin fibrosis: clinical results," Ultrasound Med. Biol., vol. 33, pp. 1191-1198, 2007.

[4] J. Mamou, A. Coron, M. Hata, J. Machi, E. Yanagihara, P. Laugier, and E. J. Feleppa, "Three-dimensional high-frequency characterization of cancerous lymph nodes," Ultrasound Med Biol, vol. 36, pp. 361-375, 2010 .

[5] F. L. Lizzi, M. Greenebaum, E. J. Feleppa, M. Elbaum, and D. J. Coleman, "Theoretical framework for spectrum analysis in ultrasonic tissue characterization," J. Acoust. Soc. Am., vol. 73, pp. 1366-1373, April 1983

[6] M. F. Insana, R. F. Wagner, D. G. Brown, and T. J. Hall, "Describing small-scale structure in random media using pulse-echo ultrasound," $J$. Acoust. Soc. Am., vol. 87, pp. 179-192, 1990.

[7] M. L. Oelze, J. F. Zachary, and W. D. O'Brien, Jr., "Characterization of tissue microstructure using ultrasonic backscatter: Theory and technique for optimization using a Gaussian form factor,' J. Acoust. Soc. Am., vol. 112, pp. 1202-1211, 2002.

[8] R. F. Wagner, M. F. Insana, and D. G. Brown, "Statistical properties of radio-frequency and envelope-detected signals with applications to medical ultrasound," J Opt Soc Am A, vol. 4, no. 5, pp. 910-922, 1987.

[9] D. P. Hruska and M. L. Oelze, "Improved parameter estimates based on the homodyned K distribution," IEEE Transactions on Ultrasonics, Ferroelectrics, and Frequency Control, vol. 56, no. 11, pp. 2471-2481, Nov. 2009.

[10] A. Coron, J. Mamou, M. Hata, J. Machi, E. Yanagihara, P. Laugier, and E. J. Feleppa, "Three-dimensional segmentation of high-frequency ultrasound echo signals from dissected lymph nodes," Proceedings of the 2008 IEEE Ultrasonics Symposium, pp. 1370-1373, 2008. 